http://jmscr.igmpublication.org/home/ ISSN (e)-2347-176x ISSN (p) 2455-0450

crossref DOI: https://dx.doi.org/10.18535/jmscr/v8i12.20

\title{
Study of Clinico-Biochemical Profile of Neonatal Seizures among Neonates Admitted in NICU, King George Hospital
}

\author{
Authors \\ Dr Srikanth Kandi ${ }^{1}$, Dr Sai Sreevani Komaragiri ${ }^{2}$ \\ ${ }^{1,2}$ Post Graduates, Department of Pediatrics, Andhra Medical College, Visakhapatnam
}

\begin{abstract}
Background: Neonatal Seizures are the most frequent and distinctive clinical manifestation of neurological dysfunction in the newborn infant. Seizure Recognition and Identification of its etiology is very important as it allows to treat the seizure actively and avoid preventable morbidity, mortality and sequelae attached to them. The aim of this study is to determine the clinico-biochemical profile of neonatal seizures.

Materials and Methods: This is a prospective observational study done for one year in the Department of pediatrics, King George Hospital, Visakhapatnam from September 2019 to September 2020. A total of 125 newborns admitted to NICU (inborn+outborn) less than or equal to 28 days of age, with clinically identified seizures were included.

Results: Incidence of neonatal seizures was 3.7\%,but the incidence of seizures was more in out born babies (12.53\%) than inborn babies(2.72\%). Incidence of seizures are 7 times more often in preterm infants than term infants. Incidence of neonatal seizures in LBW babies are 4.3 times more than normal weight babies. Neonatal seizures were more common in the first 72 hours of life. The most common type of seizure was found to be Subtle Seizures. The commonest cause of neonatal seizures was found to be Hypoxic ischemic encephalopathy (39.2\%).

Conclusion: Higher incidence of Neonatal seizures was seen in neonates with maternal risk factors of which Pregnancy induced hypertension was the major cause found. Neonatal seizures are more common in preterm babies (14.31\%), Low birth weight babies (8.19\%) and male neonates .Seizures in neonates were common during the first 3 days of life (65.6\%), the commonest cause being hypoxic ischemic encephalopathy (39.2\%). Subtle seizures were the most common type of seizure. The most common biochemical abnormality was hypoglycemia followed by hypocalcemia.
\end{abstract}

\section{Introduction}

Neonatal seizures is a common neurological problem in the newborn period, and may be the only sign of a central nervous system disorder. The presence of a seizure does not constitute a diagnosis but is a symptom of an underlying central nervous system disorder due to systemic or biochemical disturbances.
Seizure is defined as a paroxysmal involuntary electrical disturbance of brain function that may manifest as abnormal motor activity, behavioral abnormality, an impairment or loss of consciousness, sensory disturbance or autonomic dysfunction $^{2}$ and can occur at any gestational age. Earlier diagnosis of seizures and its etiology can help in treating seizure actively and avoid the 
morbidity, mortality and sequelae associated with it.

Central nervous system in newborns is particularly susceptible to seizures due to combination of enhanced excitability and diminished levels of inhibitory transmitters. The Neonate is at particular risk for development of seizures because of metabolic, anoxic, structural and infectious causes, although no causes can be identified in one fourth cases. The etiology of neonatal seizures is not disease specific and the sick neonate may present with seizures due to a combination of abnormalities.

Seizures are more common in preterm infants and they differ from seizures in full term infants as the former have less well organized seizures due to immature neuroanatomical process, Therefore any abnormal, repetitive and stereotype behaviors in neonate should be evaluated as possible seizure. Subtle seizures are observed to be the most common type of seizures.

\section{Aims \& Objectives}

1. To study various clinical features of neonatal seizures

2. To study etiology of neonatal seizures

3. To study the biochemical abnormalities in neonatal seizures

4. To correlate the biochemical abnormalities with clinical features and etiology of neonatal seizures.

\section{Materials and Methods}

Study Design: It was a hospital based Prospective observational study

Study Population: All the newborns less than or equal to 28 days with clinically identifiable seizures
Duration of the Study: September 2019 to September 2020

Place of the Study: NICU, King George hospital, Visakhapatnam

Sample Size: Sample size was Decided on the basis of prevalence of neonatal seizures in our area, 125 newborns were recruited for this study All the selected newborns were subjected to the following set of investigations

1) Complete blood count

2) Blood glucose

3) Serum electrolytes

4) Cerebro spinal fluid examination

5) Blood culture and Sensitivity

6) Neuroimaging

Inclusion Criterion: All the Newborns less than equal to 28 days admitted in the NICU (inborn /out born) with clinically identified seizures.

\section{Exclusion Criteria}

1) Major life threatening malformations.

2) Failure to obtain consent from parents for the participation in study

\section{Observation and Results}

The total number of babies admitted to nicu was 3376 (inborn+outborn), out of which 125 developed neonatal seizures. The incidence was high in out born babies as compared to inborn babies (12.5\% vs $2.7 \%)$. Neonatal seizures were more common in males, preterm and low birth weight neonates and were common during the first 3 days of life. Underlying maternal risk factors were important predisposing factors for neonatal seizures, Pregnancy induced Hypertension (20\%) was the most common risk factor predisposing to neonatal seizures.

Table 1: Sex wise distribution of neonatal seizures $(n=125)$

\begin{tabular}{|l|c|c|}
\hline Gender & No of Neonates $(\mathbf{n}=\mathbf{1 2 5})$ & Percentage $(\boldsymbol{\%})$ \\
\hline Male & 70 & 56.00 \\
\hline Female & 55 & 44.00 \\
\hline Total & 125 & 100.00 \\
\hline
\end{tabular}




\section{JMSCR Vol||08||Issue||12||Page 122-127||December}

Graph 1: Onset of seizures according to age $(n=125)$

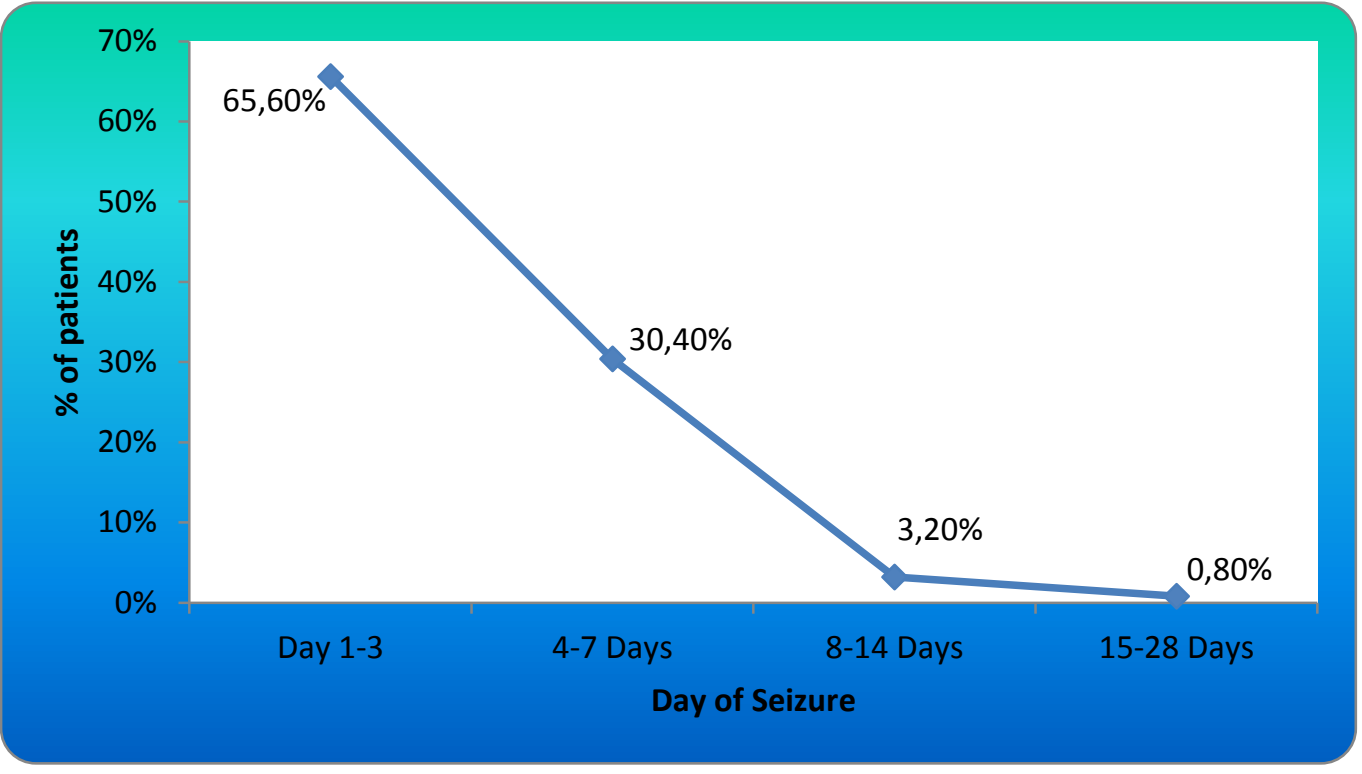

Table 3: Distribution of maternal factors and neonatal seizures $(n=125)$

\begin{tabular}{|l|c|c|}
\hline Maternal factors & $\begin{array}{c}\text { No. of neonates who developed seizures } \\
(\mathbf{n = 1 2 5})\end{array}$ & Percentage \\
\hline PIH & 25 & 20.0 \\
\hline Bleeding & 12 & 9.6 \\
\hline Premature rupture of membrane & 13 & 10.4 \\
\hline Chorioamnionitis & 7 & 5.6 \\
\hline Chronic maternal diseases & 3 & 2.4 \\
\hline Abnormal Placenta & 2 & 1.6 \\
\hline Cervical Incompetence & 1 & 0.8 \\
\hline No abnormality(Z=15.49,S) & 62 & 49.6 \\
\hline Total & 125 & 100.0 \\
\hline
\end{tabular}

The most Common type of Seizures were subtle seizures, Hypoxic ischaemic encephalopathy is the most common cause of seizures in newborn. Most common biochemical abnormality is hypoglycemia followed by hypocalcemia.32 neonates $(25.6 \%)$ were expired and the remaining 93 cases $(74.4 \%)$ were discharged

Graph 2: Distribution of neonates $(n=125)$ in different types of seizures

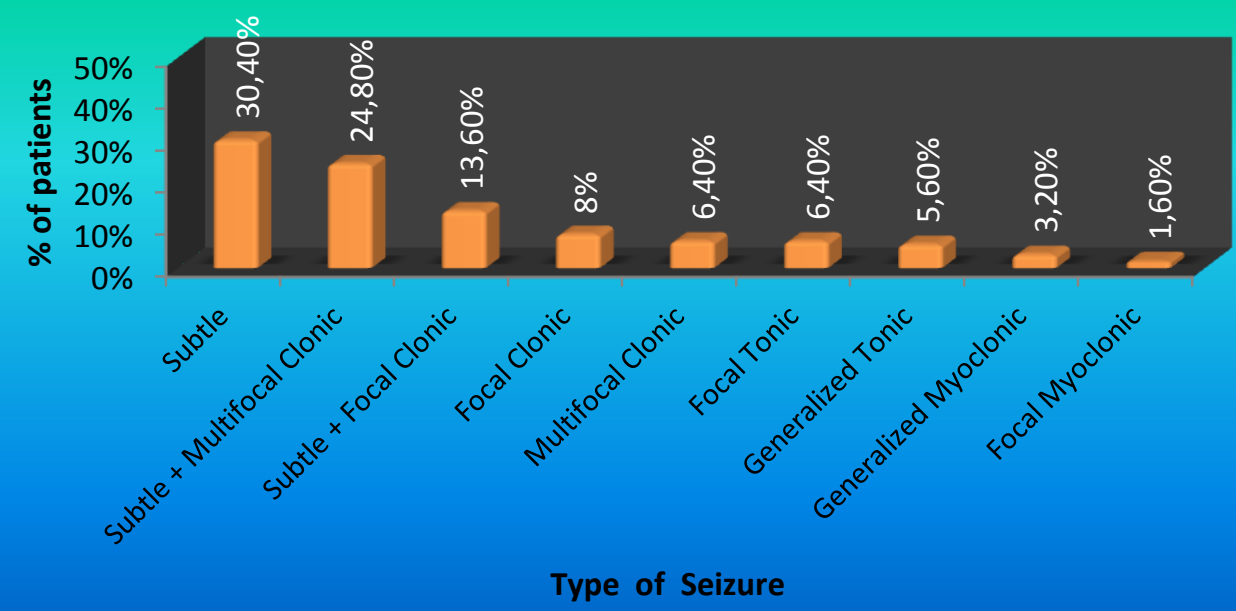


Table 4: Distribution of neonates $(n=125)$ with seizures as per etiology

\begin{tabular}{|l|c|c|}
\hline Etiology & $\begin{array}{c}\text { No of Neonates } \\
(\mathbf{n = 1 2 5})\end{array}$ & $\begin{array}{c}\text { Percentage } \\
(\mathbf{\%})\end{array}$ \\
\hline Hypoxic ischemic encephalopathy & 49 & 39.2 \\
\hline Metabolic & 26 & 20.8 \\
\hline Infection & 19 & 15.2 \\
\hline Meningitis & 17 & 13.6 \\
\hline Intra cranial Bleed & 10 & 8 \\
\hline Kernicterus & 2 & 1.6 \\
\hline Congenital malformation of brain & 2 & 1.6 \\
\hline Total & 125 & 100 \\
\hline
\end{tabular}

Table 5: Distribution of neonates with seizures as per biochemical disturbances $(n=58)$

\begin{tabular}{|l|c|}
\hline Biochemical disturbances & $\begin{array}{c}\text { No. of neonates } \\
(\mathbf{n = 5 8})\end{array}$ \\
\hline Hypoglycemia & $29(50 \%)$ \\
\hline Hypocalcemia & $17(29.3 \%)$ \\
\hline Hyponatremia & $11(18.96 \%)$ \\
\hline Hypomagnesaemia & $6(10.30 \%)$ \\
\hline
\end{tabular}

Table 6: Outcome in patients of neonatal seizures

\begin{tabular}{|l|c|c|}
\hline Outcome & $\begin{array}{c}\text { No. of neonates } \\
(\mathbf{n}=125)\end{array}$ & Percentage \\
\hline Discharge & $\mathbf{9 3}$ & $\mathbf{7 4 . 4}$ \\
\hline Death & $\mathbf{3 2}$ & $\mathbf{2 5 . 6}$ \\
\hline Total & 125 & 100 \\
\hline
\end{tabular}

\section{Discussion}

The overall incidence of the neonatal seizures in the present study period was found to be $\mathbf{3 . 7 0 \%}$ as 125 neonates developed seizures out of 3376 neonates.

The National Neonatal-Perinatal Database (NNPD) of India in the year 2002-03 that had reported an incidence of $1.0 \%$. In a study by Mohammad Kazem SABZEHEI et al in 2014 Seizures were reported in 102/1112 (9.1\%) neonatal admissions. Compared with majority of studies the incidence was high because king George hospital is a tertiary care centre to where high risk and ill newborns are often referred. In the present study of 125 neonates $70(56 \%)$ were males and 55(44\%) were females, these results are consistent with other studies. G. Sahana et al and Ruma Parvin also reported similar results in their studies.

In our study, we found the correlation of neonatal seizures with the underlying maternal factors, out of which Pregnancy induced Hypertension was the major cause found (20\%), Dr. Adeebah A.
Alyasiri et al also reported similar association of maternal risk factors and neonatal seizures.

Neonatal seizures were more common in preterm and low birth weight babies, the incidence of seizures in preterm babies is $14.3 \%$ in contrast to term babies which is $2.03 \%$. Gabriel M. Ronen et al in a study in Newfoundland found that neonatal seizures occur 6 times more often in preterm infants than in term infants. Consistent to our findings we have study done by Park $\mathbf{W}$ et al where neonatal seizures are more frequent in preterm babies. Overall incidence of neonatal seizures in low birth weight babies (63.2\%)is higher in comparison to normal weight babies (36.8\%). Shah GS and Taksande et al also reported similar numbers in their studies Among 120 neonates $82(65.6 \%)$ cases had seizures during first 3 days of life and hypoxic ischemic encephalopathy (HIE) remained the main etiological factor in 49(39.2\%) cases.

A Kumar from Varanasi, found that birth asphyxia was the commonest cause of seizure in first $48 \mathrm{hrs}$ of life which was similar to our study. 
Nawab et al study also showed $73.6 \%$ of neonatal seizures occurred within first 3 days of life

In our study subtle seizures are the most common type of seizures (30.4\%).

Dinesh Das et al also observed subtle seizures (42.6\%) as the most common type in his study, followed by tonic (33.9\%), Clonic (18.7\%). Various studies like Nawab et al, Yadav et al also showed findings similar to our study with subtle seizures as the most common type. In the present study biochemical abnormalities were observed in 58 cases out of which hypoglycemia was the commonest one (50\%) followed by Hypocalcemia (29.3\%). So hypoglycemia followed by hypocalcaemia was found in maximum percentage in our study which is comparable to the study done by Kumar.

\section{Conclusion}

The overall incidence of seizures was found to be $3.7 \%$ and there is higher incidence of neonatal seizures in outborn babies (11.94\%) than inborn babies $(2.79 \%)$. Incidence was higher in males compared to female neonates. In the present study $50.4 \%$ cases had underlying maternal factors, Pregnancy induced hypertension (PIH) is the most common one $(20 \%)$ Neonatal seizures are more common in preterm babies and low birth weight babies. Subtle seizure was the most common type of seizure found in both preterm and term neonates. Most of the neonatal seizures occur commonly during the first 3 days of life and hypoxic ischemic encephalopathy is the most common cause of seizure in neonates. The most common biochemical abnormality associated with neonatal seizures is hypoglycemia followed by hypocalcemia. High mortality rates were seen in neonatal seizures due to hypoxic ischemic encephalopathy

\section{Limitations}

The study is done in a limited number of neonates. Results may vary when done in a large number of subjects.

\section{References}

1. National Neonatal Perinatal Database. National Neonatology Forum, India. Report for year 2002-03.

2. Parvin R. Neonatal seizures: correlation between clinic-etiological profile and EEG findings. J Child Health 2014;38 (1):1923.

3. Taksande A, Vilhecar K, Jain M, Lakra M. Clinico Biochemical Profile of Neonatal Seizures. Indian J Pediatr1995;52:424-7.

4. Shah GS, Singh, Budhathoki. clinicoBiochemical profile of Neonatal seizures. JNPS 2008; 28,2:7-9.

5. Nawab T, Lakshmipathy NS,clinical profile of neonatal seizures with special reerence to biochemical abnormalities, IJCP 2016;3; 183-188.

6. Dinesh Das et al ' A study on Clinicobiochemical profile of neonatal seizures in journal of Neurology Research, 6.5-6, (2016), pg:95-101.

7. Kumar A, Gupta A, Talukdar B. Clinicoetiological and EEG profile of neonatal seizures. Indian J Pediatr. 2007 Jan;74(1): 33-7

8. Nunes, Magda Lahorgue, et al. "Neurological outcome of newborns with neonatal seizures: a cohort study in a tertiary university hospital." Arquivos de neuro-psiquiatria 66.2A (2008): 168-174

9. Cloherty, John P., and Ann R. Stark. "neonatal seizures" ,Manual of Neonatal Care. 7th ed. Philadelphia: Lippincott Williams \& Wilkins, 2004. 729-742.

10. Airede KI. Neonatal seizures and a 2 year neurological outcome. J Trop Pediatr1991; 37: 313-17.

11. Seizures neonatology, pathophysiology and management of the newborn. In: Avery GB editors. Avery's neonatology, pathophysiology and management of the newborn.10rd Ed. Philadelphia: JB Lippincott company; 2018.p.1085-1132. 
12. Mikati MA, Kliegman RM, Behrman RE, Stanton BF. Seizures in childhood; Nelson textbook of Paediatrics, 20th edition. Philadelphia.2011: WB Saunders [586]: 2013-2037.

13. Cloherty, John P., and Ann R. Stark. "neonatal seizures" ,Manual of Neonatal Care. 7th ed. Philadelphia: Lippincott Williams \& Wilkins, 2004. 729-742.

14. Meherban Singh. Chapter no 24:Metabolic disorders. In: Care of the newborn. $8^{\text {th }}$ ed. New Delhi: Sagar publication; 2015. p,373-395. 